\title{
ON THE HILBERT COEFFICIENTS, DEPTH OF ASSOCIATED GRADED RINGS AND REDUCTION NUMBERS
}

\author{
AMIR MAFI AND DLER NADERI \\ Dedicated to Professor Maria Evelina Rossi
}

\begin{abstract}
Let $(R, \mathfrak{m})$ be a $d$-dimensional Cohen-Macaulay local ring, $I$ an $\mathfrak{m}$-primary ideal of $R$ and $J=\left(x_{1}, \ldots, x_{d}\right)$ a minimal reduction of $I$. We show that if $J_{d-1}=\left(x_{1}, \ldots, x_{d-1}\right)$ and $\sum_{n=1}^{\infty} \lambda\left(I^{n+1} \cap J_{d-1}\right) /\left(J I^{n} \cap J_{d-1}\right)=i$ where $\mathrm{i}=0,1$, then $\operatorname{depth} G(I) \geq d-i-1$. Moreover, we prove that if $e_{2}(I)=$ $\sum_{n=2}^{\infty}(n-1) \lambda\left(I^{n} / J I^{n-1}\right)-2$; or if $I$ is integrally closed and

$e_{2}(I)=\sum_{n=2}^{\infty}(n-1) \lambda\left(I^{n} / J I^{n-1}\right)-3$, then $e_{1}(I)=\sum_{n=1}^{\infty} \lambda\left(I^{n} / J I^{n-1}\right)-1$, where the integers $e_{i}$ are the Hilbert coefficients of $I$. In addition, if $J$ is a minimal reduction of $I$ then we prove that the reduction number $r_{J}(I)$ is independent of $J$.
\end{abstract}

\section{INTRODUCTION}

Throughout the paper we will assume that $(R, \mathfrak{m})$ is a $d$-dimensional CohenMacaulay local ring having an infinite residue field and $I$ is an $\mathfrak{m}$-primary ideal of $R$. An ideal $J \subseteq I$ is called a reduction of $I$ if $I^{r+1}=J I^{r}$ for some nonnegative integer $r$ (see [21]). The least such $r$ is called the reduction number of $I$ with respect to $J$ and is denoted by $r_{J}(I)$. A reduction $J$ is called a minimal reduction if it does not properly contain a reduction of $I$. Under our assumption every minimal reduction is generated by a regular sequence. The reduction number of $I$ is defined as $r(I)=\min \left\{r_{J}(I): J\right.$ is a minimal reduction of $\left.I\right\}$. The reduction number $r(I)$ is said to be independent if $r(I)=r_{J}(I)$ for all minimal reductions $J$ of $I$. Sally in 27] raised the following question: If $(R, \mathfrak{m})$ is a $d$-dimensional Cohen-Macaulay local ring having an infinite residue field, then is $r(\mathfrak{m})$ independent? A natural extension of this question is to replace $r(\mathfrak{m})$ with $r(I)$. Let $G(I)=\bigoplus_{n \geq 0} I^{n} / I^{n+1}$ be the associated graded ring of $I$. Huckaba in [11] and Trung in [30] independently proved

2000 Mathematics Subject Classification. 13A30, 13D40, 13H10.

Key words and phrases. Hilbert coefficient, Minimal reduction, Associated graded ring. 
that if $\operatorname{depth} G(I) \geq d-1$, then $r(I)$ is independent(see also [19], [9], [10] and [29]). Moreover, Wu in [36] with some conditions proved that if depth $G(I) \geq d-2$, then $r(I)$ is independent. However, if $d \geq 2$ and $\operatorname{depth} G(I) \leq d-2$, then $r(I)$ is not independent in general. Counter-examples have been obtained in [11, [19] and [18].

The Hilbert function of $I$ is the numerical function $H_{I}(n)=\lambda\left(R / I^{n}\right)$ (where $\lambda($. denotes length) that measures the growth of the length of $R / I^{n}$ for all $n \geq 1$. It is well known that for $n \gg 0, H_{I}(n)$ is a polynomial in $n$ of degree $d$

$$
P_{I}(n)=\sum_{i=0}^{d}(-1)^{i} e_{i}(I)\left(\begin{array}{c}
n+d-i-1 \\
d-i
\end{array}\right)
$$

called the Hilbert polynomial of $I$, whose coefficients $e_{0}(I), e_{1}(I), \ldots, e_{d}(I)$ are uniquely determined by $I$ and called the Hilbert coefficients of $I$.

Valabrega and Valla in [31] obtained that $G(I)$ is Cohen-Macaulay if and only if there exists a minimal reduction $J$ of $I$ such that $I^{n} \cap J=I^{n-1} J$ for all $n$. Later on, Guerrieri in [5] asked that if $J$ is a minimal reduction of $I$ such that $\sum_{n \geq 1} \lambda\left(I^{n} \cap J / I^{n-1} J\right)=t$, then is $\operatorname{depth} G(I)=d-t$ ? The case $t=0$ is simply a restatement of the Valabrega-Vall theorem, whereas the case $t=1$ was proved in [5]. Some partial answers in the cases $t=2$ or $t=3$ were also proved in [6] and [8]. Huckaba and Marley in [14] and Vaz Pinto in [32] independently showed that $e_{1}(I) \leq \sum_{n \geq 1} \lambda\left(I^{n} / I^{n-1} J\right)$ and equality holds for some minimal reduction $J$ of $I$ if and only if depth $G(I) \geq d-1$. Another closely related conjecture was raised by Wang in [34] while attempting to prove Guerrieri's conjecture. Namely, he asked whether the difference $\sum_{n \geq 1} \lambda\left(I^{n} / I^{n-1} J\right)-e_{1}(I)=s \geq 0$, implies depth $G(I) \geq$ $d-s-1$. Wang first showed that an affirmative answer to his conjecture implies the validity of Guerrieri's conjecture. Then in [34] he settled the case $s=1$ (see also [23]). Unfortunately, both conjectures fail in general as shown in [35].

Corso, Polini and Rossi in [2] established a general upper bound on $e_{2}(I)$, which is reminiscent of the bound on $e_{1}(I)$ due to Huckaba and Marley in [14] and Vaz Pinto in [32]. Namely, it holds that $e_{2}(I) \leq \sum_{n \geq 2}(n-1) \lambda\left(I^{n} / I^{n-1} J\right)$ for any minimal reduction reduction $J$ of $I$. Furthermore, the upper bound is attained if and only if $\operatorname{depth} G(I) \geq d-1$. In addition, if $e_{2}(I) \geq \sum_{n \geq 2}(n-1) \lambda\left(I^{n} / I^{n-1} J\right)-2$ or if $I$ is integrally closed and $e_{2}(I) \geq \sum_{n \geq 2}(n-1) \lambda\left(I^{n} / I^{n-1} J\right)-4$, then $\operatorname{depth} G(I) \geq$ $d-2$.

In this paper we prove the following results. 
Theorem 1.1. Let $J=\left(x_{1}, \ldots, x_{d}\right)$ be a minimal reduction of $I$.

(1) Suppose that one of the following conditions holds:

(i) $e_{2}(I)=\sum_{n=2}^{\infty}(n-1) \lambda\left(I^{n} / J I^{n-1}\right)-2$;

(ii) $I$ is integrally closed and $e_{2}(I)=\sum_{n=2}^{\infty}(n-1) \lambda\left(I^{n} / J I^{n-1}\right)-3$.

Then $e_{1}(I)=\sum_{n=1}^{\infty} \lambda\left(I^{n} / J I^{n-1}\right)-1$. Moreover, we give a counterexample showing that $e_{1}(I)=\sum_{n=1}^{\infty} \lambda\left(I^{n} / J I^{n-1}\right)-1$ but the conditions (i) and (ii) do not hold.

(2) If $J_{d-1}=\left(x_{1}, \ldots, x_{d-1}\right)$ and $\sum_{n=1}^{\infty} \lambda\left(I^{n+1} \cap J_{d-1}\right) /\left(J I^{n} \cap J_{d-1}\right)=i$ where $i=0$ or $i=1$, then $\operatorname{depth} G(I) \geq d-i-1$.

Theorem 1.2. Let $J=\left(x_{1}, \ldots, x_{d}\right)$ be a minimal reduction of $I$.

(1) Suppose that one of the following conditions holds:

(i) $e_{2}(I)=\sum_{n=2}^{\infty}(n-1) \lambda\left(I^{n} / J I^{n-1}\right)-2$;

(ii) $I$ is integrally closed and $e_{2}(I)=\sum_{n=2}^{\infty}(n-1) \lambda\left(I^{n} / J I^{n-1}\right)-3$.

Then $r(I)$ is independent. Also, we study the independence of $r(I)$ with some other conditions.

For any unexplained notation or terminology, we refer the reader to [1] and [26].

\section{PRELIMINARIES}

In this section we recall some known results which is studied in [14]. An element $x \in I \backslash I^{2}$ is said to be superficial for $I$ if there is an integer $c$ such that $\left(I^{n+1}\right.$ : $x) \cap I^{c}=I^{n}$ for all $n \geq c$. If grade $I \geq 1$ and $x$ is a superficial element, then $x$ is a regular element of $R$ and by Artin-Rees Theorem $I^{n+1}: x=I^{n}$ for all $n$ sufficiently large. If $R / \mathfrak{m}$ is infinite, then a superficial element for $I$ always exists. A sequence $x_{1}, \ldots, x_{s}$ is called a superficial sequence for $I$ if $x_{1}$ is superficial for $I$ and $x_{i}$ is superficial for $I /\left(x_{1}, \ldots, x_{i-1}\right)$ for $2 \leq i \leq s$. If $J$ is a minimal reduction of $I$, then there is a superficial sequence $x_{1}, \ldots, x_{d}$ in $I$ such that $J=\left(x_{1}, \ldots, x_{d}\right)$. For any element $x \in I$ we let $x^{*}$ denote the image of $x$ in $I / I^{2}$. We note that if $x^{*}$ is a regular element of $G(I)$, then $x$ is a regular element of $R$ and $G(I /(x)) \cong G(I) /\left(x^{*}\right)$ (see [14]). 
A set of ideals $\mathcal{F}=\left\{I_{n}\right\}_{n \in \mathbb{Z}}$ of $R$ where $I_{n}=R$ for all $n \leq 0$ and $I_{1}=I$ is called a Hilbert filtration if we have $(i) I_{n+1} \subseteq I_{n}$ for all $n \geq 0$, (ii) $I_{n} I_{m} \subseteq I_{n+m}$ for all $n, m \geq 0$, and ( $i i i)$ there is a $k \geq 0$ such that $I^{n} \subseteq I_{n} \subseteq I^{n-k}$ for all $n \geq 0$. An element $x \in I_{1}$ is called superficial for $\mathcal{F}$ if there exists an integer $c$ such that $\left(I_{n+1}: x\right) \cap I_{c}=I_{n}$ for all $n \geq c$. A sequence $x_{1}, \ldots, x_{l}$ is called a superficial sequence for $\mathcal{F}$ if $x_{1}$ is superficial for $\mathcal{F}$ and $x_{i}$ is superficial for $\mathcal{F} /\left(x_{1}, \ldots, x_{i-1}\right)$ for $2 \leq i \leq l$ (see [14]). Let $\mathcal{F}$ be a Hilbert filtration and $\underline{x}=x_{1}, \ldots, x_{l} \in I_{1}$. For any integer $n$, Huckaba and Marley in [14] constructed the complex $C .(\underline{x}, \mathcal{F}, n)$ as follows: for $l=1$ they define $C .\left(x_{1}, \mathcal{F}, n\right)$ to be the complex

$$
0 \longrightarrow R / I_{n-1} \stackrel{x_{1}}{\longrightarrow} R / I_{n} \longrightarrow 0
$$

For $l>1$, assume that $C .\left(x_{1}, \ldots, x_{l-1}, \mathcal{F}, n\right)$ has been defined and consider the chain map $f: C .\left(x_{1}, \ldots, x_{l-1}, \mathcal{F}, n-1\right) \longrightarrow C .\left(x_{1}, \ldots, x_{l-1}, \mathcal{F}, n\right)$ given by multiplication by $x_{l}$. Define $C .\left(x_{1}, \ldots, x_{l-1}, \mathcal{F}, n\right)$ to be the mapping cylinder of $f$. Thus one can show that $C .\left(x_{1}, \ldots, x_{l-1}, \mathcal{F}, n\right)$ has the following form

$$
0 \longrightarrow R / I_{n-l} \longrightarrow\left(R / I_{n-l+1}\right)^{l} \longrightarrow\left(R / I_{n-l+2}\right)^{\left(\begin{array}{l}
l \\
2
\end{array}\right)} \longrightarrow \quad \ldots \quad \longrightarrow R / I_{n} \longrightarrow 0
$$

Let $C .(n)=C .\left(x_{1}, x_{2}, \ldots, x_{l}, \mathcal{F}, n\right)$ and $C^{\prime}(n)=C .\left(x_{1}, x_{2}, \ldots, x_{l-1}, \mathcal{F}, n\right)$. For any $n$ there is an exact sequence of complexes

$$
0 \longrightarrow C^{\prime}(n) \longrightarrow C_{.}(n) \longrightarrow C^{\prime}(n-1)[-1] \longrightarrow 0
$$

Thus, we have the corresponding long exact sequence on homology:

$$
\cdots \longrightarrow H_{i}\left(C_{.}^{\prime}(n)\right) \longrightarrow H_{i}(C .(n)) \longrightarrow H_{i-1}\left(C_{.}^{\prime}(n-1)\right) \stackrel{x_{l}}{\longrightarrow} H_{i-1}\left(C^{\prime}(n)\right) \longrightarrow \cdots .(*)
$$

Since $\mathcal{F}$ is a Hilbert filtration, $H_{i}(C .(\underline{x}, \mathcal{F}, n))$ has finite length for all $i$ and $n$. For $i \geq 1$, consider

$$
h_{i}(\underline{x}, \mathcal{F}):=\sum_{n=1}^{\infty} \lambda\left(H_{i}(C .(\underline{x}, \mathcal{F}, n))\right)
$$

and

$$
k_{i}(\underline{x}, \mathcal{F}):=\sum_{n=2}^{\infty}(n-1) \lambda\left(H_{i}(C .(\underline{x}, \mathcal{F}, n))\right)
$$


ON THE HILBERT COEFFICIENTS...

These integers are well-defined by [14, Lemma 3.6]. Also note that, $h_{i}(\underline{x}, \mathcal{F})=$ $k_{i}(\underline{x}, \mathcal{F})=0$ for all $i>l$. For $\underline{x}^{\prime}=x_{1}, \ldots, x_{l-1}$ we define

$$
h_{i}^{\prime}\left(\underline{x}^{\prime}, \mathcal{F}\right):=\sum_{n=1}^{\infty} \lambda\left(H_{i}\left(C .\left(\underline{x}^{\prime}, \mathcal{F}, n\right)\right)\right) .
$$

3. The associated graded Ring ANd the FIRst And SECond Hilbert COEFFICIENTS

Lemma 3.1. (114, Theorem 3.7]) Let $\mathcal{F}$ be a Hilbert filtration and $\underline{x}=x_{1}, \ldots, x_{l}$ a regular sequence on $R$ and a superficial sequence for $\mathcal{F}$. Then for each $i \geq 1$ $\sum_{j \geq i}(-1)^{j-i} h_{j}(\underline{x}, \mathcal{F}) \geq 0$. Moreover, equality occurs if and only if $\operatorname{grade}\left(\underline{x}^{*}\right) \geq$ $l-i+1$.

Lemma 3.2. Let $\mathcal{F}$ be a Hilbert filtration and $\underline{x}=x_{1}, \ldots, x_{l}$ be a regular sequence on $R$ and a superficial sequence for $\mathcal{F}$. Then, for each $i \geq 1, \sum_{j \geq i+1}(-1)^{j-i-1} k_{j}(\underline{x}, \mathcal{F}) \geq$ 0 . Moreover, equality occurs if and only if grade $\left(\underline{x}^{*}\right) \geq l-i$.

Proof. Fix $i \geq 1$ and for each $n$ let $B_{n}$ be the kernel of the map $H_{i}(C .(n)) \longrightarrow$ $H_{i-1}\left(C^{\prime}(n-1)\right)$ given in $(*)$. Then, for each $n$, we have the exact sequence

$$
0 \longrightarrow H_{l}(C .(n)) \longrightarrow \ldots \longrightarrow H_{i}\left(C^{\prime}(n-1)\right) \stackrel{x_{l}}{\longrightarrow} H_{i}\left(C^{\prime}(n)\right) \longrightarrow B_{n} \longrightarrow 0 .
$$

Therefore, for each $n$, we have

$$
\lambda\left(B_{n}\right)=\sum_{j=i+1}^{l}(-1)^{j-i-1} \lambda\left(H_{j}(C .(n))\right)+\sum_{j=i}^{l}(-1)^{j-i} \Delta\left[\lambda\left(H_{j}\left(C^{\prime}{ }^{\prime}(n)\right)\right)\right]
$$

and also by using [12, Lemma 2.7] we have

$$
\sum_{n=2}^{\infty}(n-1) \Delta\left[\lambda\left(H_{j}\left(C^{\prime} .(n)\right)\right)\right]=-\sum_{n=1}^{\infty} \lambda\left(H_{j}\left(C^{\prime} .(n)\right)\right)=-h_{j}^{\prime}\left(\underline{x}^{\prime}, \mathcal{F}\right) .
$$

Thus we see that

$$
\sum_{n=2}^{\infty}(n-1) \lambda\left(B_{n}\right)=\sum_{j=i+1}^{l}(-1)^{j-i-1} k_{j}(\underline{x}, \mathcal{F})-\sum_{j=i}^{l}(-1)^{j-i} h_{j}^{\prime}\left(\underline{x}^{\prime}, \mathcal{F}\right) .
$$

By Lemma 3.1 , we have $\sum_{j=i}^{l}(-1)^{j-i} h_{j}^{\prime}\left(\underline{x}^{\prime}, \mathcal{F}\right) \geq 0$ and so $\sum_{j=i+1}^{l}(-1)^{j-i-1} k_{j}(\underline{x}, \mathcal{F}) \geq 0$ for each $i \geq 1$.

By [14, Proposition 3.3], if grade $\left(\underline{x}^{*}\right) \geq l-i$ then $H_{j}(C .(n))=0$ for all $n$ and 
$j \geq i+1$. Thus, $k_{j}(\underline{x}, \mathcal{F})=0$ for $j \geq i+1$.

Conversely, suppose for $i \geq 1$

$$
\sum_{j=i+1}^{l}(-1)^{j-i-1} k_{j}(\underline{x}, \mathcal{F})=0 .
$$

So by $(* *), \sum_{n=2}^{\infty}(n-1) \lambda\left(B_{n}\right)=0$. Then $\sum_{n=1}^{\infty} \lambda\left(B_{n}\right)=\sum_{j=i+1}^{l}(-1)^{j-i-1} h_{j}(\underline{x}, \mathcal{F})=0$ and by Lemma 3.1 we obtain grade $\left(\underline{x}^{*}\right) \geq l-(i+1)+1=l-i$.

Following Marley [18], given a function $f: \mathbb{Z} \longrightarrow \mathbb{Z}$, define the first difference function of $f, \Delta^{1}(f): \mathbb{Z} \longrightarrow \mathbb{Z}$ by $\Delta^{1}(f)(n)=f(n+1)-f(n)$. We usually write $\Delta^{1}(f(n))$ for $\Delta^{1}(f)(n)$. Inductively define the ith difference function of $f$, $\Delta^{i}(f): \mathbb{Z} \longrightarrow \mathbb{Z}$, by $\Delta^{i}(f(n))=\Delta^{1}\left(\Delta^{i-1}(f(n))\right)$. Following Huckaba and Marley [14], a reduction of a filtration $\mathcal{F}$ is an ideal $J \subseteq I$ such that $J I_{n}=I_{n+1}$ for all large $n$. A minimal reduction of $\mathcal{F}$ is a reduction of $\mathcal{F}$ minimal with respect to containment.

Remark 3.3. By [14, §4.] we have

$$
\Delta^{d}\left[P_{\mathcal{F}}(n)-H_{\mathcal{F}}(n)\right]=\lambda\left(I_{n} / J I_{n-1}\right)-\sum_{i=2}^{d}(-1)^{i} \lambda\left(H_{i}(C .(n))\right)
$$

and

$$
e_{i}(\mathcal{F})=\sum_{n=i}^{\infty}\left(\begin{array}{c}
n-1 \\
i-1
\end{array}\right) \Delta^{d}\left[P_{\mathcal{F}}(n)-H_{\mathcal{F}}(n)\right],
$$

where $J$ is a minimal reduction of $\mathcal{F}$ and $e_{i}(\mathcal{F})$ is the Hilbert coefficients of $\mathcal{F}$. Therefore we obtain the following

$$
e_{1}(\mathcal{F})=\sum_{n=1}^{\infty} \lambda\left(I_{n} / J I_{n-1}\right)-\sum_{i=2}^{d}(-1)^{i} h_{i}(\underline{x}, \mathcal{F})
$$

and

$$
e_{2}(\mathcal{F})=\sum_{n=2}^{\infty}(n-1) \lambda\left(I_{n} / J I_{n-1}\right)-\sum_{i=2}^{d}(-1)^{i} k_{i}(\underline{x}, \mathcal{F}) .
$$

Proposition 3.4. (compare with [2, Theorem 3.1]) Let $\mathcal{F}$ be a Hilbert filtration and $J=\left(x_{1}, \ldots, x_{d}\right)$ be a minimal reduction of $\mathcal{F}$. Then

$$
e_{2}(\mathcal{F}) \leq \sum_{n=2}^{\infty}(n-1) \lambda\left(I_{n} / J I_{n-1}\right)
$$


The equality holds if and only if $\operatorname{depth} G(\mathcal{F}) \geq d-1$.

Proof. By Remark 3.3 and Lemma 3.2 we have the following

$$
e_{2}(\mathcal{F})=\sum_{n=2}^{\infty}(n-1) \lambda\left(I_{n} / J I_{n-1}\right)-\sum_{i=2}^{d}(-1)^{i-2} k_{i}(J, \mathcal{F})
$$

and

$$
\sum_{i=2}^{d}(-1)^{i-2} k_{i}(J, \mathcal{F}) \geq 0
$$

Thus

$$
e_{2}(\mathcal{F}) \leq \sum_{n=2}^{\infty}(n-1) \lambda\left(I_{n} / J I_{n-1}\right) .
$$

Also the equality follows by Lemma 3.2.

Remark 3.5. By [14, Lemma 3.2] and $(*)$ we obtain the following exact sequence

$$
\begin{gathered}
0 \longrightarrow\left(I_{n-l+1}:(\underline{x})\right) / I_{n-l} \longrightarrow\left(I_{n-l+1}:\left(\underline{x}^{\prime}\right)\right) / I_{n-l} \stackrel{x_{l}}{\longrightarrow}\left(I_{n-l+2}:\left(\underline{x}^{\prime}\right)\right) / I_{n-l+1} \longrightarrow \ldots \\
\longrightarrow\left(I_{n-1} \cap\left(\underline{x}^{\prime}\right)\right) /\left(\underline{x}^{\prime}\right) I_{n-2} \stackrel{x_{l}}{\longrightarrow}\left(I_{n} \cap\left(\underline{x}^{\prime}\right)\right) /\left(\underline{x}^{\prime}\right) I_{n-1} \longrightarrow\left(I_{n} \cap(\underline{x})\right) /(\underline{x}) I_{n-1} \longrightarrow \\
R /\left(I_{n-1},\left(\underline{x}^{\prime}\right)\right) \stackrel{x_{l}}{\longrightarrow} R /\left(I_{n},\left(\underline{x}^{\prime}\right)\right) \longrightarrow R /\left(I_{n},(\underline{x})\right) \longrightarrow 0 .
\end{gathered}
$$

If $A_{n}$ is the kernel of the map $R /\left(I_{n-1},\left(\underline{x}^{\prime}\right)\right) \stackrel{x_{l}}{\longrightarrow} R /\left(I_{n},\left(\underline{x}^{\prime}\right)\right)$, then $A_{n}=$ $\left(\left(I_{n},\left(\underline{x}^{\prime}\right)\right): x_{l}\right) /\left(I_{n-1},\left(\underline{x}^{\prime}\right)\right)$.

If $C_{n}$ is the kernel of the map $\left(I_{n} \cap(\underline{x})\right) /(\underline{x}) I_{n-1} \longrightarrow R /\left(I_{n-1},\left(\underline{x}^{\prime}\right)\right)$ or the cokernel of the map $\left(I_{n-1} \cap\left(\underline{x}^{\prime}\right)\right) /\left(\underline{x}^{\prime}\right) I_{n-2} \stackrel{x_{l}}{\longrightarrow}\left(I_{n} \cap\left(\underline{x}^{\prime}\right)\right) /\left(\underline{x}^{\prime}\right) I_{n-1}$, then $C_{n}=\left(I_{n} \cap\left(\underline{x}^{\prime}\right)\right) /\left(\left(\underline{x}^{\prime}\right) I_{n-1}+x_{l}\left(I_{n-1} \cap\left(\underline{x}^{\prime}\right)\right)\right)$. Thus if grade $\left(\underline{x}^{*}\right) \geq l-1$, then $C_{n}=0$ for all $n$ and $\left(\left(I_{n},\left(\underline{x}^{\prime}\right)\right): x_{l}\right) /\left(I_{n-1},\left(\underline{x}^{\prime}\right)\right) \cong\left(I_{n} \cap(\underline{x})\right) /(\underline{x}) I_{n-1}$.

From now on, we will assume that the filtration $\mathcal{F}=\left\{I^{n}\right\}_{n=0}^{\infty}$ is $I$-adic filtration. Let $J=\left(x_{1}, \ldots, x_{d}\right)$, where $x_{1}, \ldots, x_{d}$ is a superficial sequence in $I$, i.e., $J$ is a minimal reduction of $I$. For $i \leq d-1$, set $J_{i}=\left(x_{1}, \ldots, x_{i}\right)$ (with the convention $J_{i}=(0)$ if $i \leq 0)$, and we denote $h_{i}(\underline{x}, \mathcal{F}), k_{i}(\underline{x}, \mathcal{F})$ and $h_{i}^{\prime}\left(\underline{x}^{\prime}, \mathcal{F}\right)$ for $I$-adic filtration by $h_{i}, k_{i}$ and $h_{i}^{\prime}$, respectively. 
Proposition 3.6. Let $d \geq 2$ and $J$ be a minimal reduction of $I$ such that $\sum_{i=2}^{d}(-1)^{i-2} h_{i}=1$. Then $\operatorname{depth} G(I) \geq d-2$.

Proof. If $\sum_{i=2}^{d}(-1)^{i-2} h_{i}=1$, then by Remark $3.3, \sum_{n=0}^{\infty} \lambda\left(I^{n+1} / J I^{n}\right)-e_{1}(I)=1$ and so by [34, Theorem 3.1] we have $\operatorname{depth} G(I) \geq d-2$.

Proposition 3.7. Let $J$ be a minimal reduction of $I$ such that $\sum_{n=1}^{\infty} \lambda\left(\left(I^{n+1} \cap J_{d-1}\right) /\left(J I^{n} \cap J_{d-1}\right)\right)=0$. Then $\operatorname{depth} G(I) \geq d-1$.

Proof. By using induction on $n$, we prove that $I^{n+1} \cap J_{d-1}=J_{d-1} I^{n}$ for every $n \geq 0$. For $n=0$, there is nothing to prove. Assume that $n \geq 1$ and $I^{n} \cap J_{d-1}=J_{d-1} I^{n-1}$. From the following equalities:

$$
\begin{aligned}
I^{n+1} \cap J_{d-1} & =J I^{n} \cap J_{d-1} \\
& =\left(J_{d-1} I^{n}+x_{d} I^{n}\right) \cap J_{d-1} \\
& =J_{d-1} I^{n}+\left(x_{d} I^{n} \cap J_{d-1}\right) \\
& =J_{d-1} I^{n}+x_{d}\left(I^{n} \cap J_{d-1}\right) \\
& =J_{d-1} I^{n}+x_{d}\left(J_{d-1} I^{n-1}\right)=J_{d-1} I^{n} .
\end{aligned}
$$

The fourth equality follows from the fact that $x_{1}, x_{2}, . ., x_{d}$ form a regular sequence. Then by using Valabrega and Valla's theorem (see also [33, Theorem 5.16]) we have $x_{1}^{*}, x_{2}^{*}, \ldots, x_{d-1}^{*}$ are regular sequence and $\operatorname{depth}(G(I)) \geq d-1$.

Proposition 3.8. Let $J$ be a minimal reduction of $I$ such that $\sum_{n=1}^{\infty} \lambda\left(\left(I^{n+1} \cap J_{d-1}\right) /\left(J I^{n} \cap J_{d-1}\right)\right)=1$. Then $\operatorname{depth} G(I) \geq d-2$.

Proof. Let $B_{n+1}$ be the kernel of the map $H_{1}(C .(n+1)) \rightarrow H_{0}\left(C^{\prime}(n)\right)$ given in $(*)$. Consider the following exact sequence

$$
0 \longrightarrow H_{d}(C .(n+1)) \longrightarrow \ldots \longrightarrow H_{1}\left(C^{\prime}(n)\right) \stackrel{x_{d}}{\longrightarrow} H_{1}\left(C^{\prime}(n+1)\right) \longrightarrow B_{n+1} \longrightarrow 0 .
$$

Therefore by [14, Lemma 3.2] we have the following exact sequence

$$
\ldots \longrightarrow I^{n} \cap J_{d-1} / J_{d-1} I^{n-1} \stackrel{x_{d}}{\longrightarrow} I^{n+1} \cap J_{d-1} / J_{d-1} I^{n} \longrightarrow B_{n+1} \longrightarrow 0
$$

and $B_{n+1}=I^{n+1} \cap J_{d-1} / J I^{n} \cap J_{d-1}$. Thus

$$
\sum_{n=1}^{\infty} \lambda\left(B_{n+1}\right)=\sum_{n=1}^{\infty} \lambda\left(\left(I^{n+1} \cap J_{d-1}\right) /\left(J I^{n} \cap J_{d-1}\right)\right)=\sum_{i=1}^{d}(-1)^{i-2} h_{i}=1
$$


and so by Proposition 3.6 we have $\operatorname{depth} G(I) \geq d-2$.

Remark 3.9. Let $B_{n}$ be the kernel of the map $H_{1}(C .(n)) \rightarrow H_{0}\left(C_{.}^{\prime}(n-1)\right)$ given in $(*)$. Then for each $n$ we have the exact sequence

$$
0 \rightarrow H_{d}(C .(n)) \rightarrow \ldots \rightarrow H_{1}\left(C^{\prime}(n-1)\right) \stackrel{x_{d}}{\rightarrow} H_{1}\left(C_{.}^{\prime}(n)\right) \rightarrow B_{n} \rightarrow 0 .
$$

Therefore, for each $n$ we have

$$
\lambda\left(B_{n}\right)=\sum_{i=2}^{d}(-1)^{i-2} \lambda\left(H_{i}(C .(n))\right)+\sum_{i=1}^{d}(-1)^{i-1} \Delta\left[\lambda\left(H_{i}\left(C^{\prime}(n)\right)\right)\right] .
$$

By using [12, Lemma 2.7]

$$
\sum_{n=2}^{\infty}(n-1) \Delta\left[\lambda\left(H_{i}\left(C^{\prime} .(n)\right)\right)\right]=-\sum_{n=1}^{\infty} \lambda\left(H_{i}\left(C^{\prime} .(n)\right)\right)=-h_{i}^{\prime}
$$

and

$$
\sum_{n=1}^{\infty} \Delta\left[\lambda\left(H_{i}\left(C^{\prime} .(n)\right)\right]=0\right.
$$

Since $\sum_{n=2}^{\infty}(n-1) \lambda\left(B_{n}\right)=\sum_{i=2}^{d}(-1)^{i-2} k_{i}-\sum_{i=1}^{d}(-1)^{i-1} h_{i}^{\prime}$ and $\sum_{n=1}^{\infty} \lambda\left(B_{n}\right)$ is equal to $\sum_{i=2}^{d}(-1)^{i-2} h_{i}$, then by Lemma 3.1. $\sum_{i=1}^{d}(-1)^{i-1} h_{i}^{\prime}=0$ if and only if depth $G(I) \geq d-1$ if and only if $\sum_{n=2}^{\infty}(n-1) \lambda\left(B_{n}\right)=0$. Thus $\sum_{i=2}^{d}(-1)^{i-2} k_{i}=1$ cannot be happen. Now assume that $I$ is integrally closed ideal. Since $I^{2} \cap J=J I$ (see [17, Lemma 2.1]), then by [14, Lemma 3.2] we have $H_{1}(C .(2))=0$ and $B_{2}=0$. So if $I$ is integrally closed ideal, then $\sum_{i=2}^{d}(-1)^{i-2} k_{i}=2$ cannot happen, because in this case $\lambda\left(B_{2}\right)=1$ and this is a contradiction. So we have the following three cases:

(1) If $\sum_{i=2}^{d}(-1)^{i-2} k_{i}=2$, then we have $\sum_{i=1}^{d}(-1)^{i-1} h^{\prime}{ }_{i}=1$ and $\sum_{n=2}^{\infty}(n-1) \lambda\left(B_{n}\right)=$ 1. In this case we obtain $\lambda\left(B_{2}\right)=1$ and $\lambda\left(B_{n}\right)=0$ for any $n \neq 2$. Therefore $\sum_{n=1}^{\infty} \lambda\left(B_{n}\right)=\sum_{i=2}^{d}(-1)^{i-2} h_{i}=1$ and by Proposition 3.6 depth $G(I) \geq d-2$.

(2) If we assume $I$ to be integrally closed ideal and $\sum_{i=2}^{d}(-1)^{i-2} k_{i}=3$, then we have $\sum_{n=2}^{\infty}(n-1) \lambda\left(B_{n}\right)=1$ or 2 . The case $\sum_{n=2}^{\infty}(n-1) \lambda\left(B_{n}\right)=1$ cannot happen because 
$\lambda\left(B_{2}\right)=0$. If $\sum_{n=2}^{\infty}(n-1) \lambda\left(B_{n}\right)=2$, then $\lambda\left(B_{3}\right)=1$ and $\lambda\left(B_{n}\right)=0$ for all $n \neq 3$, so $\sum_{i=2}^{d}(-1)^{i-2} h_{i}=1$ and $\operatorname{depth} G(I) \geq d-2$.

(3) If we assume $I$ to be integrally closed ideal and $\sum_{i=2}^{d}(-1)^{i-2} k_{i}=4$, then we have $\sum_{n=2}^{\infty}(n-1) \lambda\left(B_{n}\right)=2$ or 3 . If $\sum_{n=2}^{\infty}(n-1) \lambda\left(B_{n}\right)=2$, then $\operatorname{depth} G(I) \geq d-2$. If $\sum_{n=2}^{\infty}(n-1) \lambda\left(B_{n}\right)=3$, then $\lambda\left(B_{4}\right)=1$ and $\lambda\left(B_{n}\right)=0$ for any $n \neq 4$. Thus $\sum_{i=2}^{d}(-1)^{i-2} h_{i}=1$ and $\operatorname{depth} G(I) \geq d-2$.

In the following result we compare [2, Theorem 3.1 and 3.3] with [34, Theorem 3.1].

Theorem 3.10. Let $J$ be a minimal reduction of I. Suppose that one of the following conditions holds:

(1) $e_{2}(I)=\sum_{n=2}^{\infty}(n-1) \lambda\left(I^{n} / J I^{n-1}\right)-2$;

(2) $I$ is integrally closed and $e_{2}(I)=\sum_{n=2}^{\infty}(n-1) \lambda\left(I^{n} / J I^{n-1}\right)-i$, where $i=3,4$. Then $e_{1}(I)=\sum_{n=1}^{\infty} \lambda\left(I^{n} / J I^{n-1}\right)-1$.

Proof. (1) If $e_{2}(I)=\sum_{n=2}^{\infty}(n-1) \lambda\left(I^{n} / J I^{n-1}\right)-2$, then $\sum_{i=2}^{d}(-1)^{i-2} k_{i}=2$ and by Remark $3.9 \sum_{n=1}^{\infty} \lambda\left(B_{n}\right)=\sum_{i=2}^{d}(-1)^{i-2} h_{i}=1$. Therefore by Remark 3.3 we have $e_{1}(I)=\sum_{n=1}^{\infty} \lambda\left(I^{n} / J I^{n-1}\right)-1$.

(2) If I is integrally closed and $e_{2}(I)=\sum_{n=2}^{\infty}(n-1) \lambda\left(I^{n} / J I^{n-1}\right)-i$ where $i=3,4$ then $\sum_{i=2}^{d}(-1)^{i-2} k_{i}=3$ or 4 . Therefore by Remark $3.9 \sum_{n=1}^{\infty} \lambda\left(B_{n}\right)=\sum_{i=2}^{d}(-1)^{i-2} h_{i}=1$ and so $e_{1}(I)=\sum_{n=1}^{\infty} \lambda\left(I^{n} / J I^{n-1}\right)-1$.

The following example shows that the converse of Theorem 3.10 in general is not true. 
Example 3.11. Let $R=k[x, y]_{(x, y)}$, where $k$ is a field and $I=\left(x^{6}, y^{6}, x^{5} y+x^{2} y^{4}\right)$. Then by using Macaulay 2 [4] we obtain the following Hilbert polynomial

$$
P_{I}(n)=36\left(\begin{array}{c}
n+1 \\
2
\end{array}\right)-15\left(\begin{array}{l}
n \\
1
\end{array}\right)+11
$$

and $e_{1}(I)=\sum_{n=1}^{\infty} \lambda\left(I^{n} / J I^{n-1}\right)-1$ but $e_{2}(I)=\sum_{n=2}^{\infty}(n-1) \lambda\left(I^{n} / J I^{n-1}\right)-3$ and $I$ is not integrally closed.

\section{The Depth of ASsociated GRADED Ring AND The REDUCtion Number}

Let $A=\bigoplus_{m \geq 0} A_{m}$ be a Notherian graded ring where $A_{0}$ is an Artinian local ring, $A$ is generated by $A_{1}$ over $A_{0}$ and $A_{+}=\bigoplus_{m>0} A_{m}$. Let $H_{A_{+}}^{i}(A)$ denote the i-th local cohomology module of $A$ with respect to the graded ideal $A_{+}$and set $a_{i}(A)=\max \left\{m \mid\left[H_{A_{+}}^{i}(A)\right]_{m} \neq 0\right\}$ with the convention $a_{i}(A)=-\infty$, if $H_{A_{+}}^{i}(A)=0$. The Castelnuovo-Mumford regularity is defined by $\operatorname{reg}(A):=\max \left\{a_{i}(A)+i \mid i \geq 0\right\}$. In the following theorem, for simplicity, we use $a_{i}$ instead of $a_{i}(G(I))$.

Theorem 4.1. Let $J$ denote a minimal reduction of $I$. Suppose that one of the following conditions holds:

(1) $e_{2}(I)=\sum_{n=2}^{\infty}(n-1) \lambda\left(I^{n} / J I^{n-1}\right)-2$;

(2) I is integrally closed and $e_{2}(I)=\sum_{n=2}^{\infty}(n-1) \lambda\left(I^{n} / J I^{n-1}\right)-3$.

Then $r(I)$ is independent.

Proof. (1) By Remark 3.9, $\sum_{i=2}^{d}(-1)^{i-2} k_{i}=2$ and depth $G(I) \geq d-2$. If $\operatorname{depth} G(I) \geq$ $d-1$, then by [18, Theorem 2] $r_{J}(I)=\operatorname{reg}(G(I))$ and so the result in this case follows. Now we assume that $\operatorname{depth} G(I)=d-2$ and $\sum_{i=2}^{d}(-1)^{i-2} k_{i}=2$. By Remark 3.9, $\lambda\left(B_{2}\right)=1$ and $\lambda\left(B_{n}\right)=0$ for any $n \neq 2$. Therefore we have $\lambda\left(I^{2} \cap J_{d-1} / J_{d-1} I\right)=1$, $\lambda\left(I^{n} \cap J_{d-1} / J_{d-1} I^{n-1}\right)=0$ for any $n \neq 2$, $\lambda\left(\left(I^{2}+J_{d-2}\right): x_{d-1} / I\right)=1$ and $\lambda\left(\left(I^{n}+J_{d-2}\right): x_{d-1} / I^{n-1}+J_{d-2}\right)=0$ for any $n \neq$ 2. If depth $G(I)=d-2$, then by applying [19, Theorem 2.1] there are two cases:

(i) If $a_{d-1} \leq a_{d}$, then $r_{J}(I)=a_{d}+d=\operatorname{reg}(G(I))$.

(ii)If $a_{d}<a_{d-1}$, then $r_{J}(I) \leq a_{d-1}+d-1=\operatorname{reg}(G(I))$ and $a_{d-1}=\max \left\{n \mid\left(I^{n+d-1}+\right.\right.$ $\left.\left.J_{d-2}\right): x_{d-1} \neq I^{n+d-2}+J_{d-2}\right\}$. Therefore by the above process $a_{d-1}=3-d$ and so 
for any reduction $J$ of $I, r_{J}(I) \leq 2$. Hence $r(I)$ is independent.

(2) Let $i=3$. Then by Remark 3.9, $\lambda\left(B_{3}\right)=1$ and $\lambda\left(B_{n}\right)=0$ for any $n \neq 3$. Thus

$$
\lambda\left(I^{3} \cap J_{d-1} / J_{d-1} I^{2}\right)=1=\lambda\left(\left(I^{3}+J_{d-2}\right): x_{d-1} / I^{2}\right)
$$

and

$$
\lambda\left(I^{n} \cap J_{d-1} / J_{d-1} I^{n-1}\right)=0=\lambda\left(\left(I^{n}+J_{d-2}\right): x_{d-1} / I^{n-1}+J_{d-2}\right)
$$

for any $n \neq 3$. If $\operatorname{depth} G(I)=d-2$, then by [19, Theorem 2.1] there are two cases:

(i) If $a_{d-1} \leq a_{d}$, then $r_{J}(I)=a_{d}+d=\operatorname{reg}(G(I))$.

(ii) If $a_{d}<a_{d-1}$, then $r_{J}(I) \leq a_{d-1}+d-1=\operatorname{reg}(G(I))$ and $a_{d-1}=\max \left\{n \mid\left(I^{n+d-1}+\right.\right.$ $\left.\left.J_{d-2}\right): x_{d-1} \neq I^{n+d-2}+J_{d-2}\right\}$. Therefore by the above process $a_{d-1}=4-d$ and so for any reduction $J$ of $I, r_{J}(I) \leq 3$. Since $I$ is integrally closed, then $r(I)$ is independent.

Let $\mathfrak{a}$ be an ideal of grade at least 1 in a Noetherian ring $R$. The Ratliff-Rush closure of $\mathfrak{a}$ is defined as the ideal $\widetilde{\mathfrak{a}}=\cup_{n \geq 1}\left(\mathfrak{a}^{n+1}: \mathfrak{a}^{n}\right)$. It is a refinement of the integral closure of $\mathfrak{a}$ and $\widetilde{\mathfrak{a}}=\mathfrak{a}$ if $\mathfrak{a}$ is integrally closed (see [24]).

Proposition 4.2. (compare with $[2$, Theorem 3.3(b)]) Let $d=3$ and $J$ be a minimal reduction of $I$. If $\widetilde{I}=I$ and $e_{2}(I)=\sum_{n=2}^{\infty}(n-1) \lambda\left(I^{n} / J I^{n-1}\right)-3$ then $\operatorname{depth} G(I) \geq 1$.

Proof. If $e_{2}(I)=\sum_{n=2}^{\infty}(n-1) \lambda\left(I^{n} / J I^{n-1}\right)-3$, then $\sum_{i=2}^{d}(-1)^{i-2} k_{i}=3$. Hence by Remark 3.9, $\sum_{i=1}^{d}(-1)^{i-1} h_{i}^{\prime}=1$ and $\sum_{n=2}^{\infty}(n-1) \lambda\left(B_{n}\right)=2$. Therefore we have the following cases:

1) $\lambda\left(B_{3}\right)=1$ and $\lambda\left(B_{n}\right)=0$ for any $n \neq 3$.

or

2) $\lambda\left(B_{2}\right)=2$ and $\lambda\left(B_{n}\right)=0$ for any $n \neq 2$.

For the first case, by Proposition 3.6. $\operatorname{depth} G(I) \geq 1$ and the result follows. Let us consider the second case. Since $\widetilde{I}=I$, we obtain that $I^{2}: x=I$ for every superficial element $x$. Since $\lambda\left(B_{2}\right)=2$, it follows $\lambda\left(I^{2} \cap J_{2} / J_{2} I\right)=2$. It therefore follows $\lambda\left(I^{2} \cap J_{1} / J_{1} I\right)=0$. Hence $\sum_{i=1}^{d}(-1)^{i-1} h_{i}^{\prime} \geq 2$ and this is a contradiction.

Northcott in [20] proved that $e_{1}(I) \geq e_{0}(I)-\lambda(R / I)$ and after that Huneke in [15] showed that $e_{1}(I)=e_{0}(I)-\lambda(R / I)$ if and only if $I^{2}=J I$. When this is the case, 
$G(I)$ is Cohen-Macaulay. Sally in [28] proved that if $d \geq 2, e_{1}(I)-e_{0}(I)+\lambda(R / I)=1$ and $e_{2}(I) \neq 0$, then $\operatorname{depth} G(I) \geq d-1$ (see also [14, Corollary 4.15] and [7, Proposition 3.1]). Also Itoh in [16] with this conditions proved that if $I$ is integrally closed, then $G(I)$ is Cohen-Macaulay. In the following example we show that the integrally closedness of $I$ is essential for the Cohen-Macaulayness of $G(I)$.

The following example appears in [2].

Example 4.3. Let $R=k[x, y, z]_{(x, y, z)}$, where $k$ is a field, and $I=\left(x^{2}-y^{2}, y^{2}-\right.$ $\left.z^{2}, x y, y z, x z\right)$. Then, by Macaulay 2 , we have $e_{0}(I)=8, e_{1}(I)=4, e_{2}(I)=0$ and $\operatorname{depth} G(I)=0$.

Lemma 4.4. Let $J$ be a minimal reduction of $I$. If $e_{1}(I)-e_{0}(I)+\lambda(R / I)=2$ and $I=\bar{I}$, then $\operatorname{depth} G(I) \geq d-1$.

Proof. By using the Sally machine and the good behaviour of $e_{1}(I)$ modulo superficial elements (see [3, Proposition 1.2] and [14, Lemma 2.2]), we may reduce the statement to dimension two. If $e_{1}(I)-e_{0}(I)+\lambda(R / I)=2$, by [25, Corollary 1.5] we have $r_{J}(I) \leq 3$ for any minimal reduction $J$ of $I$. If there exist a minimal reduction $J$ of $I$ such that $r_{J}(I)=2$, then by [17, Lemma 2.1] $G(I)$ is CohenMacaulay. If for any minimal reduction $J$ of $I, r_{J}(I)=3$ then by [25, Remark 1.8] $r_{J}(I) \leq e_{1}(I)-e_{0}(I)+\lambda(R / I)+2-\lambda\left(I^{2} / J I\right)$. Hence $r(I)=3 \leq 4-\lambda\left(I^{2} / J I\right)$ and $\lambda\left(I^{2} / J I\right) \leq 1$ and so by [25, Corollary 1.7] depth $G(I) \geq 1$.

Proposition 4.5. Let $J$ be a minimal reduction of I. If $e_{1}(I)-e_{0}(I)+\lambda(R / I) \leq 3$ and $I=\bar{I}$, then $r(I)$ is independent.

Proof. By Lemma 3.15 and the above explanation, we can assume $e_{1}(I)-e_{0}(I)+$ $\lambda(R / I)=3$ and also by [22, Corollary 4.7] and [11, Lemma 1.1] we may assume that $d=2$. If $\operatorname{depth} G(I) \geq 1$, then by [19, Corollary 2.2] we have $r_{J}(I)=\operatorname{reg}(G(I))$ and so $r(I)$ is independent. Now we may assume that $\operatorname{depth} G(I)=0$. Since $e_{1}(I)-e_{0}(I)+\lambda(R / I)=3$, by [25, Corollary 1.5] $r_{J}(I) \leq 4$ for any minimal reduction $J$ of $I$. If for some $J, r_{J}(I)=2$ then by [17, Lemma 2.1] $G(I)$ is Cohen-Macaulay and this is a contradiction with $\operatorname{depth} G(I)=0$. If for some $J, r_{J}(I)=4$ then by [25, Remark 1.8] $r_{J}(I) \leq e_{1}(I)-e_{0}(I)+\lambda(R / I)+2-\lambda\left(I^{2} / J I\right)$. Therefore $\lambda\left(I^{2} / J I\right) \leq 1$ and so depth $G(I) \geq 1$ and this is also a contradiction. Hence we can assume that for any minimal reduction $J$ of $I, r_{J}(I)=3$ and so $r(I)$ is independent. 
Corollary 4.6. Let $J$ be a minimal reduction of $I$. If $e_{1}(I)-e_{0}(I)+\lambda(R / I) \leq$ $r_{J}(I)-1, I=\bar{I}$ and $\operatorname{depth} G(I) \geq d-2$, then $r(I)$ is independent.

Proof. By using the Sally machine and the good behaviour of $e_{1}(I)$ modulo superficial elements, we may reduce the statement to dimension two. If $e_{1}(I)-e_{0}(I)+$ $\lambda(R / I) \leq r_{J}(I)-1$, then by [25, Remark 1.8] $r_{J}(I) \leq e_{1}(I)-e_{0}(I)+\lambda(R / I)+2-$ $\lambda\left(I^{2} / J I\right)$. Hence $\lambda\left(I^{2} / J I\right) \leq 1$ and so $\operatorname{depth} G(I) \geq d-1$. Therefore the result follows by [18, Theorem 2].

Example 4.7. Let $R=k[x, y]_{(x, y)}$ where $k$ is a field, and $I=\left(x^{6}, y^{6}, x^{5} y, x^{3} y^{3}, x^{2} y^{4}, x y^{5}\right)$. Then by Macaulay 2 we have $e_{0}(I)=36, e_{1}(I)=15$ and $\lambda(R / I)=22$. Hence $e_{1}(I)-e_{0}(I)+\lambda(R / I)=1$ but $\operatorname{depth} G(I)=0$ and $r_{J}(I)=2=\operatorname{reg}(G(I))$ for all minimal reduction $J$ of $I$.

Example 4.8. Let $R=k[x, y]_{(x, y)}$ where $k$ is a field, and $I=\left(x^{6}, y^{6}, x^{5} y, x^{3} y^{3}, x^{2} y^{4}\right)$. Then by Macaulay 2 we have $e_{0}(I)=36, e_{1}(I)=15$ and $\lambda(R / I)=23$. Hence $e_{1}(I)-e_{0}(I)+\lambda(R / I)=2$ but $\operatorname{depth} G(I)=0$ and $r_{J}(I)=2=\operatorname{reg}(G(I))$ for all minimal reduction $J$ of $I$.

Example 4.9. Let $R=k[x, y]_{(x, y)}$ where $k$ is a field, and $I=\left(x^{6}, y^{6}, x^{5} y, x^{3} y^{3}, x y^{5}\right)$. Then by Macaulay 2 we have $e_{0}(I)=36, e_{1}(I)=15$ and $\lambda(R / I)=23$. Hence $e_{1}(I)-e_{0}(I)+\lambda(R / I)=3$ but $\operatorname{depth} G(I)=0$ and $r_{J}(I)=2=\operatorname{reg}(G(I))$ for all minimal reduction $J$ of $I$.

Example 4.10. Let $R=k[x, y]_{(x, y)}$ where $k$ is a field, and $I=\left(x^{6}, y^{6}, x^{5} y, x^{2} y^{4}, x y^{5}\right)$. Then by Macaulay 2 we have $e_{0}(I)=36, e_{1}(I)=15$ and $\lambda(R / I)=24$. Hence $e_{1}(I)-e_{0}(I)+\lambda(R / I)=3$ but for two minimal reduction $J_{1}=\left(x^{6}, x^{5} y+y^{6}\right)$ and $J_{2}=\left(x^{6}, y^{6}\right)$ we have $r_{J_{1}}(I)=2$ and $r_{J_{2}}(I)=3$ and $\operatorname{depth} G(I)=0$ because $I$ is not integrally closed.

The following example is due to Huckaba and Huneke [13].

Example 4.11. Let $R=k[x, y, z]_{(x, y, z)}$ where $k$ is a field of characteristic $\neq 3$. Let $\mathfrak{a}=\left(x^{4}, x\left(y^{3}+z^{3}\right), y\left(y^{3}+z^{3}\right), z\left(y^{3}+z^{3}\right)\right)$ and set $I=\mathfrak{a}+\mathfrak{m}^{5}$ where $\mathfrak{m}$ is the maximal ideal of $R$. The ideal $I$ is a integral closer $m$-primary ideal whose associated graded ring $\operatorname{gr}_{I}(R)$ has depth 2. We checked that $e_{0}(I)=76, e_{1}(I)=48$ and $\lambda(R / I)=31$ so $e_{1}(I)-e_{0}(I)+\lambda(R / I)=3$ and $r(I)$ is independent.

Acknowledgement. We would like to thank deeply grateful to the referee for the careful reading of the manuscript and the helpful suggestions 


\section{REFERENCES}

[1] W. Bruns and J. Herzog, Cohen-Macaulay rings, Cambridge University Press, Cambridge, UK, (1998).

[2] A. Corso, C. Polini and M. E. Rossi, Depth of associated graded rings via Hilbert coeffcients of ideals, J. Pure and Appl. Algebra, 201 (2005), 126-141.

[3] J. Elias, M. E. Rossi and G. Valla, On the coefficients of the Hilbert polynomial, J. Pure and Appl. Algebra, 108 (1996), 35-60

[4] D. R. Grayson and M. E. Stillman, Macaulay 2, a software system for research in algebraic geometry, Available at http:// www.math.uiuc.edu/Macaulay2.

[5] A. Guerrieri, On the depth of the associated graded ring of an m-primary ideal of a CohenMacaulay local ring, J. Algebra, 167 (1994), 745-757.

[6] A. Guerrieri, On the depth of the associated graded ring, Proc. Amer. Math. Soc., 123 (1995), $11-20$.

[7] A. Guerrieri and M. E. Rossi, Hilbert coefficients of Hilbert filtrations, J. Algebra, 199 (1998), 40-61.

[8] A. Guerrieri and M. E. Rossi, Estimates on the depth of the associated graded ring, J. Algebra, 211 (1999), 457-471.

[9] L. T. Hoa, Reduction numbers and rees algebras of powers of an ideal, Proc. Amer. Math. Soc., 119 (1993), 415-422.

[10] L. T. Hoa, Reduction numbers of equimultiple ideals, J. Pure and Appl. Algebra, 109 (1996), 111-126.

[11] S. Huckaba, Reduction numbers of ideals of higher analytic spread, Proc. Camb. Phil. Soc., 102 (1987), 49-57.

[12] S. Huckaba, A d-dimensional extension of a lemma of Huneke's and formulas for the Hilbert coefficients, Proc. Amer. Math. Soc., 124 (1996), 1393-1401.

[13] S. Huckaba and C. Huneke, Normal ideals in regular rings, J. Reine Angew. Math., 510 (1999), 63-82.

[14] S. Huckaba and T. Marley, Hilbert coefficients and the depths of associated graded rings, J. London Math. Soc., 56 (1997), 64-76.

[15] C. Huneke, Hilbert functions and symbolic powers, Michigan Math. J.,34 (1987), 293-318.

[16] S. Itoh, Hilbert coefficients of integrally closed ideals, J. Algebra, 176 (1995), 638-652.

[17] A. Mafi and D. Naderi, A note on reduction numbers and Hilbert-Samuel functions of ideals over Cohen-Macaulay rings, Turkish J. Math., 40 (2016), 766-769.

[18] T. Marley, The coefficients of the Hilbert polynomial and the reduction number of an ideal, J. London Math. Soc., 40 (1989), 1-8.

[19] T. Marley, The reduction number of an ideal and the local cohomology of the associated graded ring, Proc. Amer. Math. Soc., 117 (1993), 335-341.

[20] D. G. Northcott, A note on the coefficients of the abstract Hilbert function, J. London Math. Soc., 35 (1960) 209-214. 
[21] D. G. Northcott and D. Rees, Reduction of ideals in local rings, Proc. Cambridge Philos. Soc., 50 (1954), 145-158.

[22] K. Ozeki and M. E. Rossi, The structure of the Sally module of integrally closed ideals, Nagoya Math. J., 227 (2017), 49-76.

[23] C. Polini, A filtration of the Sally module and the associated graded ring of an ideal, Comm. Algebra, 28 (2000), 1335-1341.

[24] L. J. Ratliff and D. Rush, Two notes on reductions of ideals, Indiana Univ. Math. J., 27 (1978), 929-934.

[25] M. E. Rossi, A bound on the reduction number of a primary ideal, Proc. Amer. Math. Soc., 128 (1999), 1325-1332.

[26] M. E. Rossi and G. Valla, Hilbert functions of filtered modules, Lecture Notes of the Unione Matematica Italiana, Vol. 9, Springer-Verlag, Berlin; UMI, Bologna, 2010.

[27] J. D. Sally, Reductions, local cohomology and Hilbert functions of local ring. In Commutative Algebra: Durham 1981. London Math. Soc. Lecture Notes Series no. 72(Cambridge University Press, 1982), pp. 231-241.

[28] J. D. Sally, Hilbert coefficients and reduction number 2, J. Algebraic Geom., 1 (1992), 325-333.

[29] B. Strunk Castelnuovo-Mumford regularity, postulation numbers, and reduction numbers, J. Algebra, 311(2007), 538-550.

[30] N. V. Trung, Reduction exponent and dgree bound for the defining equations of graded rings, Proc. Amer. Math. Soc., 101 (1987), 229-236.

[31] P. Valabrega and G. Valla, Form rings and regular sequence, Nagoya Math. J., 72 (1978), 93-101.

[32] M. Vaz Pinto, Hilbert functions and Sally modules, J. Algebra, 192 (1997), 504-523.

[33] J. K. Verma, Hilbert coefficients and depth of the associated graded ring of an ideal, arXiv:0801.4866

[34] H. J. Wang, Hilbert coefficients and the associated graded rings, Proc. Amer. Math. Soc., 128 (2000) 963-973.

[35] H. J. Wang, On the associated graded rings of ideals of reduction 2, J. Algebra, 236 (2001), 192-215.

[36] Y. Wu, Reduction numbers and Hilbert polynomials of ideals in higher dimensional CohenMacaulay local rings, Math. Proc. Camb. Phil. Soc., 111 (1992), 47-56.

A. Mafi, Department of Mathematics, University of Kurdistan, P.O. Box: 416, SANANDAJ, IRAN.

E-mail address: a_mafi@ipm.ir

D. Naderi, Department of Mathematics, University of Kurdistan, P.O. Box: 416, SANANDAJ, IRAN.

E-mail address: dler.naderi65@gmail.com 